\title{
Research Article \\ On the Absolute Sum of Chromatic Polynomial Coefficient of Graphs
}

\author{
Shubo Chen \\ Department of Mathematics, Hunan City University, Yiyang, Hunan 413000, China \\ Correspondence should be addressed to Shubo Chen, shubochen@gmail.com
}

Received 1 December 2010; Accepted 9 February 2011

Academic Editor: Dalibor Froncek

Copyright (C) 2011 Shubo Chen. This is an open access article distributed under the Creative Commons Attribution License, which permits unrestricted use, distribution, and reproduction in any medium, provided the original work is properly cited.

The absolute sum of chromatic polynomial coefficient of forest, $q$-tree, unicyclic graphs, and quasiwheel graphs, are determined in this paper.

\section{Introduction}

For a century ago, one of the most famous problems in mathematics was to prove the FourColor Problem. During the period that the Four-Color Problem was unsolved, which spanned more than a century, many approaches were introduced with the hopes that they would lead to a solution of this famous problem. In 1913, Birkhoff [1] defined a function $P(M, \lambda)$ that gives the number of proper $\lambda$-colorings of a map $M$ for a positive integer $\lambda$. As we will see, $P(M, \lambda)$ is a polynomial in $\lambda$ for every map $M$ and is called the chromatic polynomial of $M$. Consequently, if it could be verified that $P(M, 4)>0$ for every map $M$, then this would have established the truth of the Four-Color Conjecture. In 1932, Whitney [2] expanded the study of chromatic polynomials from maps to graphs. While Whitney obtained a number of results on chromatic polynomials of graphs and others obtained results on the roots of chromatic polynomials of planar graphs, this did not contribute to a proof of the Four-Color Conjecture. Renewed interest in chromatic polynomials of graphs occurred in 1968 when Read [3] wrote a survey paper on chromatic polynomials. Let $G$ be a graph and $\lambda \in \mathbb{N}$. A mapping $f$ : $V(G) \rightarrow\{1,2, \ldots, \lambda\}$ is called a $\lambda$-coloring of $G$ if $f(u) \neq f(v)$ whenever the vertices $u$ and $v$ are adjacent in $G$. For a positive integer $\lambda$, the number of different proper $\lambda$-colorings of $G$ is denoted by $P(G, \lambda)$ and is called the chromatic polynomial of $G$. By convention, $P(G, 0)=0$, and $P(G, \lambda) \geq 1$ if and only if $G$ is $\lambda$-colorable. More precisely, we have

$$
\chi(G)=\min \{\lambda \in \mathbb{N}: P(G, \lambda) \geq 1\} .
$$


There are many results about the coefficient of the chromatic polynomials of graphs, see related references [4-8]. In [5], the authors characterized the absolute sum of chromatic polynomial of trees, 2-trees, cycles, wheels and completed graphs, and obtained the sharp upper and lower bounds for the absolute sum of chromatic polynomial coefficients of any connected graphs. In this paper, we investigate absolute sum of chromatic polynomial coefficients of forest, $q$-tree, unicyclic graphs and quasiwheel graphs.

\section{Preliminaries}

Let $G=(V, E)$ be a graph whose sets of vertices and edges are $V(G)$ and $E(G)$, respectively, $n=|V(G)|, m=|E(G)|$ are the number of vertices and edges of $G$. $E^{\prime} \subseteq E(G)$, we denote by $G-E^{\prime}\left(G+E^{\prime}\right)$ the subgraph of $G$ obtained by deleting (adding) the edges of $E^{\prime} . W \subseteq V(G)$, $G-W(G+W)$ denote the subgraph of $G$ obtained by deleting (adding) the vertices of $W$ and the edges incident with them. $G \cdot x y$ is the graph obtained from $G$ by contracting $x$ and $y$ and removing any loop and all but one of the multiple edges, if they arise, where $x, y \in V(G)$. Terminologies and notations not defined here can be found in [9]. The following basic results will be used and can be found in the references cited.

Lemma 2.1 (see [9]). (i) Let $x$ and $y$ be two nonadjacent vertices in a graph $G$. Then $P(G, \lambda)=$ $P(G+x y, \lambda)+P(G \cdot x y, \lambda)$.

(ii) Let $e \in E(G)$, then $P(G, \lambda)=P(G-e, \lambda)-P(G \cdot e, \lambda)$.

(iii) For the empty graph $O_{n}$ of order $n$, it is clear that $P\left(O_{n}, \lambda\right)=\lambda^{n}$. More generally, if $G=\bigcup_{i=1}^{k} G_{i}$, then

$$
P(G, \lambda)=\prod_{i=1}^{k} P\left(G_{i}, \lambda\right) .
$$

Lemma 2.2 (see [5]). (i) Let $G$ be an arbitrary graph with $n$ vertices, then the sum of chromatic polynomial of $P(G, \lambda)=\sum_{i=1}^{n} a_{i} \lambda^{i}$ is

$$
\sum_{i=1}^{n} a_{i}= \begin{cases}0, & m \neq 0 \\ 1, & m=0\end{cases}
$$

(ii) Let $T$ be an arbitrary tree with $n$ vertices and $P(T, \lambda)=\sum_{i=1}^{n} a_{i} \lambda^{i}=\lambda(\lambda-1)^{n-1}$. Then, $\sum_{i=1}^{n}\left|a_{i}\right|=2^{n-1}$.

(iii) Let $T_{n}^{2}$ be an arbitrary 2-tree with $n$ vertices and $P\left(T_{n}^{2}, \lambda\right)=\sum_{i=1}^{n} a_{i} \lambda^{i}=\lambda(\lambda-1)(\lambda-2)^{n-2}$. Then, $\sum_{i=1}^{n}\left|a_{i}\right|=2 \cdot 3^{n-2}$.

(iv) Let $C_{n}$ be an arbitrary graph with $n$ vertices and $P\left(C_{n}, \lambda\right)=\sum_{i=1}^{n} a_{i} \lambda^{i}=(\lambda-1)^{n}+$ $(-1)^{n}(\lambda-1)$. Then, $\sum_{i=1}^{n}\left|a_{i}\right|=2^{n}-2$.

(v) Let $W_{n}$ be an arbitrary graph with $n$ vertices and $P\left(W_{n}, \lambda\right)=\sum_{i=1}^{n} a_{i} \lambda^{i}=\lambda(\lambda-2)^{n-1}+$ $(-1)^{n-1} \lambda(\lambda-2)$. Then, $\sum_{i=1}^{n}\left|a_{i}\right|=3^{n-1}-3$. 
(vi) Let $K_{n}$ be an arbitrary graph with $n$ vertices and $P\left(K_{n}, \lambda\right)=\sum_{i=1}^{n} a_{i} \lambda^{i}=\lambda(\lambda-1)(\lambda-$ 2) $\cdots(\lambda-n+1)$. Then, $\sum_{i=1}^{n}\left|a_{i}\right|=\prod_{i=1}^{n} i$.

then

(vii) Let $G$ be an arbitrary connected graph with $n(n>1)$ vertices and $P(G, \lambda)=\sum_{i=1}^{n} a_{i} \lambda^{i}$,

$$
2^{n-1} \leq \sum_{i=1}^{n}\left|a_{i}\right| \leq \prod_{i=1}^{n} i
$$

The left equality holds if and only if $G \cong T_{n}$ and the right equality holds if and only if $G \cong K_{n}$.

Given $q \in \mathbb{N}$, the class of $q$-trees $T_{n}^{q}$ is defined recursively as follows: any complete graph $K_{q}$ is a $q$-tree, and any $q$-tree of order $n+1$ is a graph obtained from a q-tree $G$ of order $n$, where $n \geq q$, by adding a new vertex and joining it to each vertex of $a K_{q}$ in $G$. As an example, a 3-tree is depicted in Figure 1(a).

Lemma 2.3 (see [9]). Let $T_{n}^{q}$ is a q-tree with $n$ vertices, then

$$
P\left(T_{n}^{q}, \lambda\right)=\lambda(\lambda-1) \cdots(\lambda-q+1)
$$

Lemma 2.4 (see [6]). Let $\mathfrak{U}_{n}(m)$ is a unicyclic graph order $n$ and girth $m$, then

$$
P\left(\mathcal{U}_{n}(m), \lambda\right)=(\lambda-1)^{n}+(-1)^{m}(\lambda-1)^{n-m+1} .
$$

For $1 \leq s \leq n-2$, we denote by $W(n, s)$ the graph obtained from $W_{n}$ by deleting all but s consecutive spokes. For convenience, we call $W(n, s)$ as the quasiwheel graphs, as shown in Figure 1(b).

Lemma 2.5 (see [9]). Let $W(n, s)$ is a quasiwheel graph with $n$ vertices, then

$$
P(W(n, s), \lambda)=(\lambda-2)^{s-1}\left[(\lambda-1)^{n-s+1}+(-1)^{n-s}\right]+(-1)^{n-1} \lambda(\lambda-2) .
$$

\section{Main Results}

In this section, we investigate the absolute sum of chromatic polynomial coefficients of forests, $q$-trees, unicyclic graphs, and quasiwheel graphs.

Theorem 3.1. Let $F$ be the forest of order $n$ and $c$ components and $P(F, \lambda)=\sum_{i=1}^{n} a_{i} \lambda^{i}$. Then $\sum_{i=1}^{n}\left|a_{i}\right|=2^{n-c}$.

Proof. It is a direct result of the combination of Lemma 2.1(iii) and Lemma 2.2(ii).

Theorem 3.2. Let $T_{n}^{q}$ is a q-tree of order $n(n \geq q)$ and $P\left(T_{n}^{q}, \lambda\right)=\sum_{i=1}^{n} a_{i} \lambda^{i}$. Then $\sum_{i=1}^{n}\left|a_{i}\right|=$ $q ! \times(q+1)^{n-q}$. 


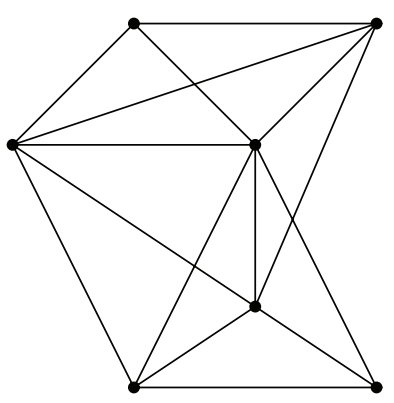

(a) 3-tree

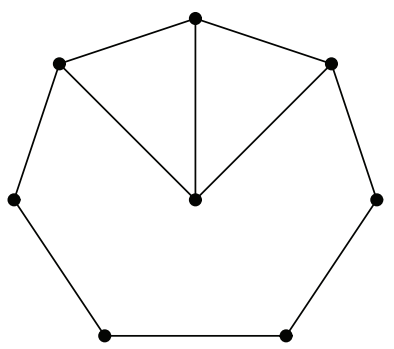

(b) $W(8,3)$

Figure 1: The graphs 3-tree and $W(8,3)$.

Proof. We prove the result inductively on $n$.

(i) $n=q$. In this case $T_{n}^{q} \cong K_{q}$.

$$
P\left(T_{q}^{q}, \lambda\right)=\lambda(\lambda-1) \cdots(\lambda-q+1)=\sum_{i=1}^{v} a_{i} \lambda^{i}
$$

then $\sum_{i=1}^{v}\left|a_{i}\right|=\prod_{i=1}^{q} i=q$ !.

(ii) Assume that $n=k(k \geq q)$, let $P\left(T_{k}^{q}, \lambda\right)=\sum_{i=1}^{k} b_{i} \lambda^{i}$, we have $\sum_{i=1}^{k}\left|b_{i}\right|=q ! \times(q+1)^{k-q}$.

(iii) When $n=k+1$, let $P\left(T_{k+1}^{q}, \lambda\right)=\sum_{i=1}^{k+1} c_{i} \lambda^{i}$. $u$ is a vertex with degree $q$, and the edges connecting $u$ are $e_{1}, e_{2}, \ldots, e_{q}$, respectively. By Lemma 2.1(ii), we have

$$
\begin{aligned}
P\left(T_{k+1}^{q}, \lambda\right) & =P\left(T_{k+1}^{q}-e_{1}, \lambda\right)-P\left(T_{k+1}^{q} \cdot e_{1}, \lambda\right) \\
& =P\left(\left(T_{k+1}^{q}-e_{1}\right)-e_{2}, \iota\right)-P\left(\left(T_{k+1}^{q}-e_{1}\right) \cdot e_{2}, \lambda\right)-P\left(T_{k+1}^{q} \cdot e_{1}, \lambda\right) \\
& =\cdots \\
& =\lambda P\left(T_{k}^{q}, \lambda\right)-q P\left(T_{k}^{q}, \lambda\right) \\
& =\lambda \sum_{i=1}^{k} b_{i} \lambda^{i}-q \sum_{i=1}^{k} b_{i} \lambda^{i} .
\end{aligned}
$$

Then, $\sum_{i=1}^{k+1}\left|c_{i}\right|=\sum_{i=1}^{k}\left|b_{i}\right|+q \sum_{i=1}^{k}\left|b_{i}\right|=(q+1) \times q ! \times(q+1)^{k-q}=q ! \times(q+1)^{k+1-q}$.

Combining above, the results follows.

Theorem 3.3. Let $\mathcal{U}_{n}(m)$ is a unicyclic graph order $n$ and girth $m$ and $P\left(\mathcal{U}_{n}(m), \lambda\right)=\sum_{i=1}^{n} a_{i} \lambda^{i}$. Then, $\sum_{i=1}^{n}\left|a_{i}\right|=2^{n}-2^{n-m+1}$. 
Proof. From Lemma 2.3, we have

$$
\begin{aligned}
P\left(\mathcal{U}_{n}(m), \lambda\right) & =(\lambda-1)^{n}+(-1)^{m}(\lambda-1)^{n-m+1} \\
& =\sum_{i=0}^{n} C_{n}^{i} \lambda^{n-i}(-1)^{i}+(-1)^{m} \sum_{i=0}^{n-m+1} C_{n-m+1}^{i} \lambda^{n-m+1-i}(-1)^{i} \\
& =\sum_{i=0}^{m-2} C_{n}^{i} \lambda^{n-i}(-1)^{i}+\sum_{i=m-1}^{n}\left[C_{n}^{i}(-1)^{i}+C_{n-m+1}^{i+1-m}(-1)^{i+1}\right] \lambda^{n-i} .
\end{aligned}
$$

Let $(\lambda-1)^{n}+(-1)^{m}(\lambda-1)^{n-m+1}=\sum_{i=1}^{n} a_{i} \lambda^{i}$, then

$$
\begin{aligned}
\sum_{i=0}^{n}\left|a_{i}\right| & =\sum_{i=0}^{m-2}\left|C_{n}^{i}\right|+\sum_{i=m-1}^{n}\left|C_{n}^{i}-C_{n-m+1}^{i+1-m}\right| \\
& =\sum_{i=0}^{m-2} C_{n}^{i}+\sum_{i=m-1}^{n}\left|C_{n}^{n-i}-C_{n-m+1}^{n-i}\right| \\
& =\sum_{i=0}^{m-2} C_{n}^{i}+\sum_{i=m-1}^{n} C_{n}^{n-i}-\sum_{i=m-1}^{n} C_{n-m+1}^{n-i} \\
& =2^{n}-2^{n-m+1} .
\end{aligned}
$$

Theorem 3.4. Let $W(n, s)$ is a quasiwheel graph with $n$ vertices and $P(W(n, s), \lambda)=\sum_{i=1}^{n} a_{i} \lambda^{i}$. Then, $\sum_{i=1}^{n}\left|a_{i}\right|=3^{s-1}\left(2^{n-s+1}-1\right)-3$.

Proof. From Lemma 2.5, we have

$$
\begin{aligned}
P(W(n, s), \lambda)= & (\lambda-2)^{s-1}\left[(\lambda-1)^{n-s+1}+(-1)^{n-s}\right]+(-1)^{n-1} \lambda(\lambda-2) \\
= & {\left[\lambda^{s-1}+\cdots+C_{s-1}^{1} \lambda(-2)^{s-2}+(-2)^{s-1}, \lambda^{n-s+1}+\cdots+C_{n-s+1}^{2} \lambda^{2}(-1)^{n-s-1}\right.} \\
& \left.\quad+C_{n-s+1}^{1} \lambda(-1)^{n-s}\right]+(-1)^{n-1}\left(\lambda^{2}-2 \lambda\right) \\
= & \lambda^{n}+\cdots+\left[C_{s-1}^{1} 2^{s-2} C_{n-s+1}^{1}(-1)^{n-2}+C_{n-s+1}^{2} 2^{s-1}(-1)^{n-2}+(-1)^{n-1}\right] \lambda^{2} \\
& +\left[C_{n-s+1}^{1} 2^{s-1}(-1)^{n-1}+2(-1)^{n}\right] \lambda .
\end{aligned}
$$

Let $(\lambda-2)^{s-1}\left[(\lambda-1)^{n-s+1}+(-1)^{n-s}\right]=\sum_{i=1}^{n} b_{i} \lambda^{i}$, then $\sum_{i=1}^{n}\left|b_{i}\right|=3^{s-1}\left(2^{n-s+1}-1\right)$. Moreover,

$$
\begin{aligned}
\sum_{i=1}^{n} b_{i} \lambda^{i}= & \lambda^{n}+\cdots+\left[C_{s-1}^{1} 2^{s-2} C_{n-s+1}^{1}(-1)^{n-2}+C_{n-s+1}^{2} 2^{s-1}(-1)^{n-2}\right] \lambda^{2} \\
& +C_{n-s+1}^{1} 2^{s-1}(-1)^{n-1} \lambda .
\end{aligned}
$$


Thus,

$$
\begin{aligned}
\sum_{i=1}^{n}\left|b_{i}\right| & =\sum_{i=3}^{n}\left|b_{i}\right|+C_{s-1}^{1} 2^{s-2} C_{n-s+1}^{1}+C_{n-s+1}^{2} 2^{s-1}+C_{n-s+1}^{1} 2^{s-1} \\
\sum_{i=1}^{n}\left|a_{i}\right| & =\sum_{i=3}^{n}\left|b_{i}\right|+C_{s-1}^{1} 2^{s-2} C_{n-s+1}^{1}-1+C_{n-s+1}^{2} 2^{s-1}+C_{n-s+1}^{1} 2^{s-1}-2 \\
& =3^{s-1}\left(2^{n-s+1}-1\right)-3 .
\end{aligned}
$$

This completes the proof.

\section{Acknowledgment}

Researches are supported by the Education Bureau of Hunan Province, China (Grant no. 10B015).

\section{References}

[1] G. D. Birkhoff, "The Reducibility of Maps," American Journal of Mathematics, vol. 35, no. 2, pp. 115-128, 1913.

[2] H. Whitney, "The coloring of graphs," Annals of Mathematics. Second Series, vol. 33, no. 4, pp. 688-718, 1932.

[3] R. C. Read, "An introduction to chromatic polynomials," Journal of Combinatorial Theory, Series A, vol. 4, pp. 52-71, 1968.

[4] H. Whitney, "Congruent graphs and the connectivity of graphs," American Journal of Mathematics, vol. 54, no. 1, pp. 150-168, 1932.

[5] N. Liu, "Research on the sum of chromatic coefficients," Operation Transactions, vol. 7, pp. 67-74, 2003 (Chinese).

[6] G. Haggard and T. R. Mathies, "Using thresholds to compute chromatic polynomials," Ars Combinatoria, vol. 58, pp. 85-95, 2001.

[7] E. J. Farrell, "On the derivative of the chromatic polynomial," Bulletin of the Institute of Combinatorics and Its Applications, vol. 29, pp. 33-38, 2000.

[8] F. Van Bussel, C. Ehrlich, D. Fliegner, S. Stolzenberg, and M. Timme, "Chromatic polynomials of random graphs," Journal of Physics, vol. 43, no. 17, Article ID 175002, 2010.

[9] F. M. Dong, K. M. Koh, and K. L. Teo, Chromatic Polynomials and Chromaticity of Graphs, World Scientific, Hackensack, NJ, USA, 2005. 


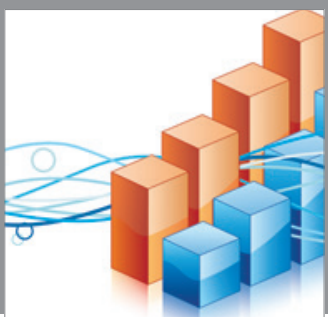

Advances in

Operations Research

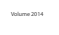

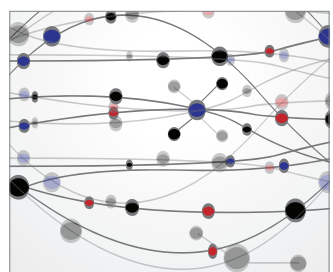

\section{The Scientific} World Journal
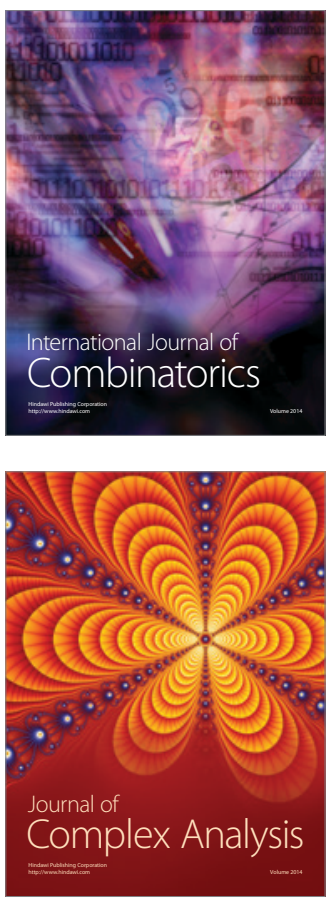

International Journal of

Mathematics and

Mathematical

Sciences
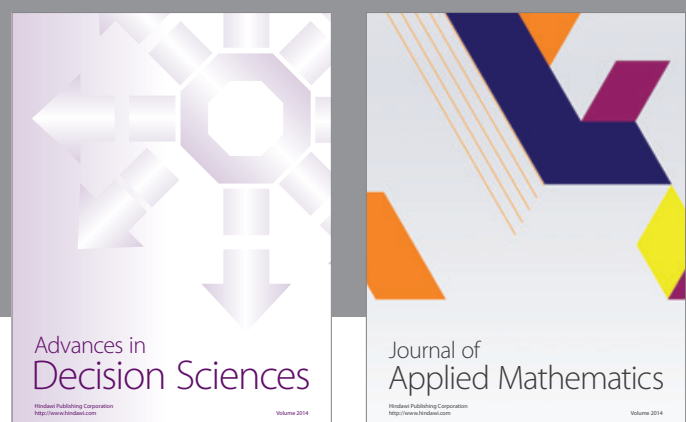

Journal of

Applied Mathematics
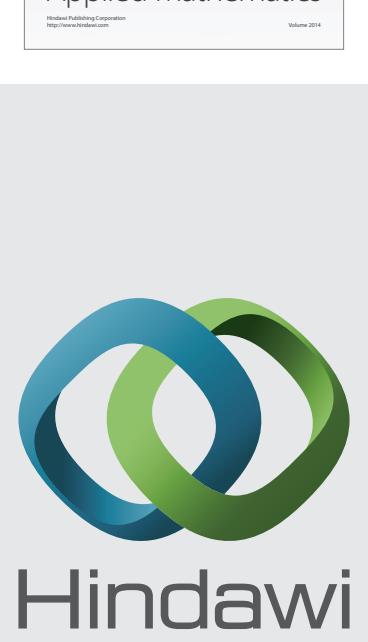

Submit your manuscripts at http://www.hindawi.com
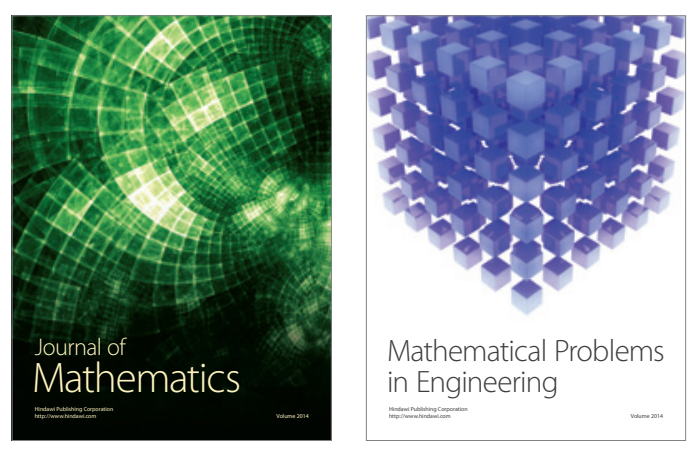

Mathematical Problems in Engineering
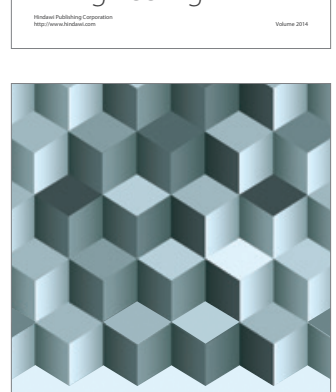

Journal of

Function Spaces
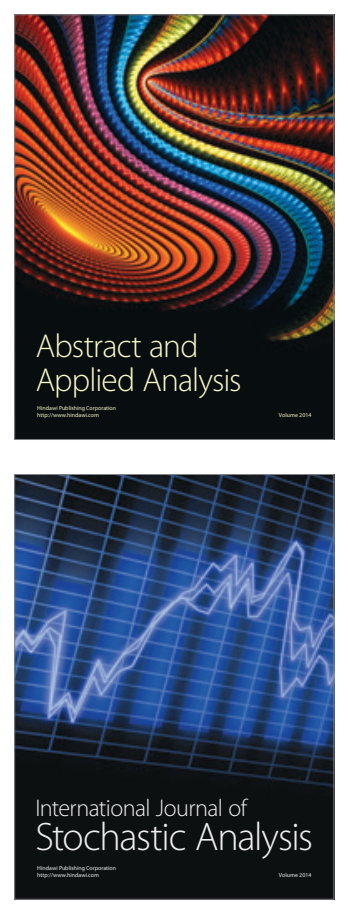

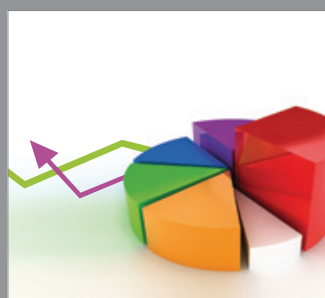

ournal of

Probability and Statistics

Promensencen
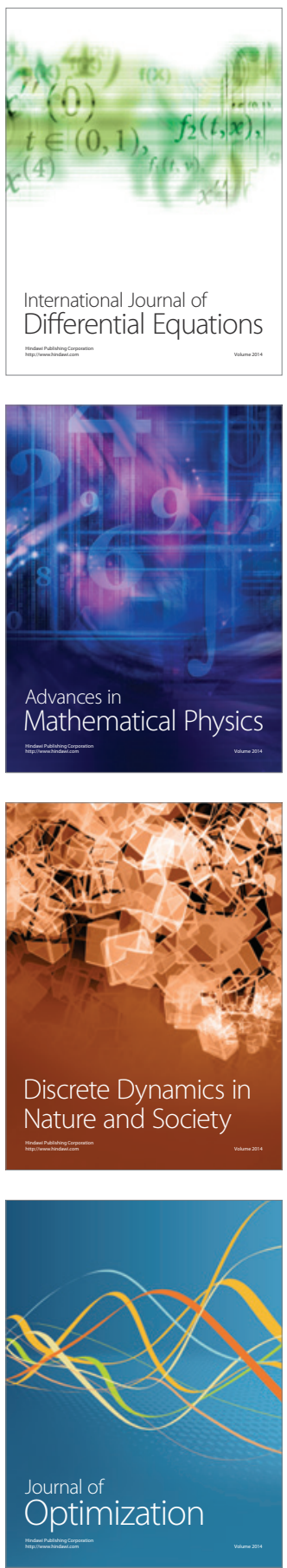